\title{
ENTRELACEMENTS DE SEMI-GROUPES PROVENANT DE PAIRES DE GELFAND
}

\author{
Philippe Biane ${ }^{1}$
}

\begin{abstract}
Résumé. On donne des exemples d'entrelacements entre semi-groupes markoviens obtenus au moyen de considérations de théorie des groupes sur les paires de Gelfand.
\end{abstract}

Classification Mathématique. 60J23, 60J35, 22D15.

Reçu le 11 décembre 2009.

\section{INTRODUCTION}

La notion d'entrelacement de noyaux markoviens, ou de semi-groupes de tels noyaux, a fait l'objet de plusieurs travaux de Carmona et al. [6] (dont Yor), et Hirsch et Yor [13]. Elle s'est révélée très utile dans plusieurs problèmes concernant les processus stochastiques, on pourra voir par exemple [9], [14] et [15] pour plusieurs développements récents. Deux noyaux markoviens $P(u, \mathrm{~d} x)$ et $Q(v, \mathrm{~d} y)$ sur deux espaces $X$ et $Y$ sont dits entrelacés par un noyau $\Lambda(v, \mathrm{~d} x)$ sur $Y \times X$, si on a

$$
\Lambda P=Q \Lambda
$$

Deux semi-groupes de noyaux markoviens sont entrelacés si cette relation a lieu, avec le même $\Lambda$, pour tous les temps. L'exemple le plus connu d'entrelacement est sans doute celui du mouvement Brownien et du processus de Bessel de dimension 3, qui joue un rôle important dans le théorème de Pitman [17]. Le but de cet article est de donner quelques nouveaux exemples intéressants d'entrelacements de processus, obtenus par des considérations de théorie des groupes que j'ai déjà évoquées auparavant [3]. Afin de motiver ces considérations je vais tout d'abord décrire dans ce langage le cas bien connu des processus de Bessel. On peut obtenir un entrelacement entre deux semi-groupes par couplage, en réalisant les deux processus de Markov que l'on cherche à entrelacer comme projections d'un processus de Markov sur un espace plus gros. On considère ainsi un mouvement Brownien de dimension $d$. Si on regarde une de ses coordonnées, on obtient un mouvement Brownien unidimensionnel, tandis que si l'on observe sa norme, on obtient un processus de Bessel (de dimension $d$ ). On peut définir deux applications naturelles. La première associe à une fonction sur $\mathbf{R}$ une fonction sur $\mathbf{R}^{d}$ : à $f$ définie sur $\mathbf{R}$ correspond la fonction $f\left(x_{1}\right)$ qui ne dépend que de la première coordonnée. La seconde prend une fonction sur $\mathbf{R}^{d}$ et calcule sa moyenne sous l'action du groupe des rotations de $\mathbf{R}^{d}$. On obtient ainsi une fonction radiale, que l'on considère comme fonction sur $\mathbf{R}_{+}$. La composée de ces deux applications transforme donc une fonction sur $\mathbf{R}$ en une fonction sur $\mathbf{R}_{+}$et elle est donnée par un noyau markovien $\Lambda$, qui entrelace les semi-groupes du

Mots Clés. Entrelacement de semi-groupes de noyaux markoviens, paires de Gelfand.

1 CNRS, France. Philippe.Biane@univ-mlv.fr 
mouvement Brownien et du processus de Bessel, soit en notant $P_{t}$ le semi-groupe du mouvement Brownien et $R_{t}^{d}$ celui processus de Bessel de dimension $d$,

$$
\Lambda P_{t}=R_{t}^{d} \Lambda
$$

En fait le noyau markovien se calcule très facilement, il s'agit du noyau

$$
\Lambda(r, \mathrm{~d} x)=\frac{1}{r^{d-1} v_{d-1}}\left(r^{2}-x^{2}\right)^{(d-3) / 2} \mathrm{~d} x \quad r \geq 0 ; \quad-r<x<r
$$

$v_{d-1}$ étant le volume de la sphère unité dans $\mathbf{R}^{d}$; cette mesure de probabilité est la loi de la première coordonnée d'un point tiré au hasard sur une sphère de rayon $r$, avec la loi uniforme.

Plus généralement, supposons que $P_{t}$ et $Q_{t}$ sont des semi-groupes sur des espaces $X$ et $Y$ et que l'on dispose d'un couplage, c'est-à-dire un processus $\mathcal{Z}$ sur un espace $Z$, de semi-groupe $S_{t}$, qui admet les deux processus que l'on cherche à entrelacer comme marginales. Autrement dit, il existe des applications

$$
u: Z \rightarrow X, \quad v: Z \rightarrow Y
$$

telles que $u\left(\mathcal{Z}_{t}\right)$ soit un processus de Markov de semi-groupe $P_{t}$ et $v\left(\mathcal{Z}_{t}\right)$ un processus de Markov de semi-groupe $Q_{t}$. Si on désigne encore par $u$ et $v$ les applications qui à une fonction $\operatorname{sur} X$ (ou $Y$ ) associent la fonction $f \circ u$ (ou $f \circ v$ ) sur $Z$, on a alors les relations

$$
u \circ P_{t}=S_{t} \circ u \quad v \circ Q_{t}=S_{t} \circ v .
$$

On ne peut en général en tirer plus. Le point crucial dans la construction de l'entrelacement entre Bessel et Brownien est qu'il existe un groupe compact $K$ (le groupe des rotations dans le cas du Bessel) agissant sur l'espace $Z$, sous l'action duquel le semi-groupe est invariant. Notons $I_{K}$ l'application

$$
f \mapsto \int_{K} f \circ k \mathrm{~d} k
$$

de moyennisation par rapport à l'action de $K$, où $\mathrm{d} k$ désigne la mesure de Haar sur $K$. Une fonction invariante par l'action de $K$ sur $Z$ s'identifie avec une fonction sur l'espace des orbites $Z / K$. Si $Y \sim Z / K$ et si

$$
I_{K} \circ S_{t}=Q_{t} \circ I_{K}
$$

alors on déduit de (1.1) et (1.2) que le noyau $\Lambda=u \circ I_{K}$ entrelace les semi-groupes $P_{t}$ et $Q_{t}$

$$
\Lambda P_{t}=Q_{t} \Lambda .
$$

Dans [3] j'ai proposé une version non commutative de cette construction, c'est-à-dire la construction d'un 《 couplage non commutatif » entre deux semi-groupes sur des espaces adéquats, qui permet d'obtenir des entrelacements remarquables. Je vais la rappeler dans la partie suivante, puis donner les exemples.

\section{UNE CONSTRUCTION DE THÉORIE DES GROUPES}

Soit $G$ un groupe localement compact et $K$ un sous-groupe compact. Le groupe $K$ agit par translations à droite et à gauche sur l'ensemble des mesures boréliennes signées, finies, sur $G$. Notons $M(G)$ l'ensemble de ces mesures. C'est une algèbre pour le produit de convolution. On note $M^{K}(G)$ le sous-ensemble des mesures qui sont biinvariantes par $K$, autrement dit, qui vérifient

$$
\mu=\delta_{k_{1}} * \mu * \delta_{k_{2}}
$$


pour tous $k_{1}, k_{2} \in K, \delta_{k}$ étant la masse de Dirac en $k$ et $*$ le produit de convolution. C'est une sous-algèbre de $M(G)$, et on dit que $(G, K)$ est un couple de Gelfand si elle est commutative. L'étude des couples de Gelfand est un chapitre important de l'analyse harmonique et de la théorie des représentations, voir par exemple [10] pour un exposé introductif.

On considère maintenant un semi-groupe multiplicatif de fonctions de type positif sur $G$. Je donnerai des exemples de tels semi-groupes en temps discret et en temps continu. En temps discret un tel semi-groupe est donné par les puissances positives $\varphi^{n}$ d'une fonction continue, de type positif $\varphi$ sur $G$, biinvariante par $K$ et telle que $\varphi(e)=1$. En temps continu, on prendra $\psi$ une fonction sur $G$, continue, conditionnellement de type négatif, biinvariante par $K$, telle que $\psi(0)=0$. Elle engendre alors un semi-groupe multiplicatif $\exp (t \psi) ; t \geq 0$ formé de fonctions de type positif. Un tel semi-groupe, en temps discret ou continu, agissant par multiplication sur $M(G)$, induit un semi-groupe d'applications complètement positives, $Z_{t}$, de $M(G)$ dans lui-même.

La notion d'application complètement positive vient de la théorie des algèbres d'opérateurs, il s'agit d'un analogue non commutatif de la notion de noyau markovien. Une étude, dans un cadre probabiliste, de cette notion se trouve dans le livre [16]. Toutefois il n'y a pas besoin de savoir précisément ce que signifie ce terme pour comprendre la suite. La seule chose que nous utiliserons est le fait que si une application complètement positive envoie une $C^{*}$-algèbre commutative dans une $C^{*}$-algèbre commmutative, alors elle est donnée par un noyau markovien sur les spectres de ces algèbres.

Le semi-groupe $Z_{t}$ est l'analogue du semi-groupe sur le « gros » espace $Z$ de l'introduction, l'espace $Z$ luimême n'existe pas, ou plutôt il existe en tant qu'« espace non commutatif » au sens de [8]. On va maintenant décrire les espaces $X$ et $Y$ de l'introduction. Considérons tout d'abord un sous-groupe commutatif fermé $T$ de $G$. Les mesure portées par $T$ forment une sous-algèbre commutative de $M(G)$, isomorphe à $M(T)$. On peut complèter l'algèbre $M(G)$ au moyen d'une norme de $C^{*}$-algèbre, en prenant

$$
\|\mu\|=\sup _{\pi}\left|\int_{G} \pi(g) \mathrm{d} \mu(g)\right|
$$

où le supremum est pris sur les représentation unitaires continues du groupe $G$. Le semi-groupe $Z_{t}$ se prolonge par continuité à cette complétion. La complétion de $M(T)$ est alors une $C^{*}$-algèbre commutative qui, d'après le théorème de Gelfand-Naimark, admet un spectre, i.e. est isomorphe à l'algèbre des fonctions continues et nulles à l'infini sur un espace topologique localement compact $s p(T)$. En fait ici, par la théorie de Fourier, $s p(T)$ n'est autre que $\hat{T}$, le groupe dual de $T$. La restriction de la fonction $\exp \left(t \psi\right.$ ) (ou de $\varphi^{n}$ en temps discret) à $T$ est la transformée de Fourier d'une mesure de probabilité sur $\hat{T}$, et ces mesures de probabilité, pour $t \geq 0$ forment un semi-groupe de convolution sur $\hat{T}$, que nous noterons $P_{t}$. Ici l'indice $t$ est discret ou continu, suivant le cas. Le processus correspondant est donc une marche aléatoire sur le dual de $T$. L'espace $\hat{T}$ est ici notre espace $X$, il reste à exhiber l'espace $Y$. Comme $(G, K)$ est une paire de Gelfand, la sous-algèbre $M^{K}(G)$ est commutative. Sa complétion est de nouveau isomorphe à l'algèbre des fonctions continues et nulles à l'infini sur un espace topologique localement compact $\Omega$, le spectre de la paire de Gelfand. En fait $\Omega$ s'identifie avec l'ensemble des fonctions sphériques de type positif de la paire $(G, K)$, c'est-à-dire les fonctions continues, de type positif $\varphi$ sur $G$, qui sont biinvariantes par $K$ et qui définissent des caractères de l'algèbre $M^{K}(G)$ par la formule $\mu \rightarrow \mu(\varphi)$. C'est l'ensemble $\Omega$ qui sera l'espace $Y$. Comme la fonction $\psi$ est biinvariante par $K$, la multiplication par $\exp (t \psi)$ envoie $M^{K}(G)$ sur elle-même, et préserve la positivité (au sens des opérateurs), elle correspond donc à un noyau markovien sur le spectre $\Omega$ de la paire de Gelfand. Ce noyau markovien $Q_{t}(\omega, \mathrm{d} \xi)$ est caractérisé par l'égalité

$$
\exp (t \psi) \varphi_{\omega}=\int_{\Omega} \varphi_{\xi} Q_{t}(\omega, \mathrm{d} \xi)
$$

qui exprime la décomposition de la fonction de type positif $\exp (t \psi) \varphi_{\omega}$ en combinaison convexe de fonctions sphériques. 
On dispose d'une projection de $M(G)$ sur $M^{K}(G)$, qui est donnée par la moyenne de l'action de $K$, soit

$$
I_{K}: M(G) \rightarrow M^{K}(G) ; \quad \mu \mapsto \int_{K \times K} \delta_{k_{1}} * \mu * \delta_{k_{2}} \mathrm{~d} k_{1} \mathrm{~d} k_{2}
$$

Par construction, cette projection est un opérateur complètement positif, et sa restriction à l'algèbre commutative $M(T)$, définit un noyau $\Lambda(\tau, \mathrm{d} \omega)$ sur $\hat{T} \times \Omega$. Comme l'opération de multiplication par une fonction biinvariante commute à la convolution par $K$ on voit que ce noyau entrelace les deux semi-groupes $P_{t}$ et $Q_{t}$.

J'ai décrit dans [3] comment cette construction, appliquée au groupe d'Heisenberg, donne un entrelacement entre le processus de Yule et le processus de Bessel. Je vais considérer dans la suite de cet article d'autres classes de groupes.

\section{EXEMPLES}

\subsection{Paires de Gelfand et actions doublement transitives}

Nous allons dans la suite considérer trois types d'exemples. Tout d'abord dans la section suivante nous envisagerons les groupes compacts, et en particulier le groupe $S U(2)$. Puis, dans les sections suivantes, des groupes agissant sur les arbres, que j'ai déjà traités en partie dans [4], et enfin le groupe $S L_{2}(\mathbf{C})$ agissant sur l'espace hyperbolique.

Ces deux derniers exemples sont des cas particuliers de groupes agissant de façon doublement transitive sur un espace métrique. Rappelons ( $c f$. [10]) que si $G$ un groupe localement compact, agissant par isométries sur un espace métrique $X$, on dit que son action est doublement transitive si pour tous $x, y, x^{\prime}, y^{\prime} \in X$, vérifiant $\mathrm{d}(x, y)=\mathrm{d}\left(x^{\prime}, y^{\prime}\right)$, il existe $g \in G$ tel que $g x=x^{\prime}$ et $g y=y^{\prime}$. Si le sous-groupe $K$ laissant fixe un point de $G$ est compact, alors la paire $(G, K)$ est une paire de Gelfand, $c f$. [10], IV.II.

\subsection{Groupes compacts}

Soit $K$ un groupe compact. Dans le groupe $G=K \times K$, considérons le sous-groupe diagonal $(k, k), k \in K$ isomorphe à $K$. Le couple $(G, K)$ est une paire de Gelfand. Soit $\mu$ une mesure sur $K$, biinvariante par l'action de $K$, alors la projection de cette mesure sur la première composante de $K \times K$ est une mesure centrale sur $K$, c'est-à-dire une mesure invariante par conjugaison. Ainsi, l'algèbre $M^{K}(G)$ est naturellement isomorphe à l'algèbre de convolution des mesures centrales sur $K$, et la théorie des fonctions sphériques de la paire de Gelfand $K \times K$ est identique à celle des caractères du groupe $K$.

J'ai déjà étudié des chaînes de Markov construites de cette façon dans [1], [2], [5]. Je vais rappeler ici l'exemple le plus simple, celui du groupe $K=S U(2)$ des matrices unitaires $2 \times 2$ de déterminant 1 . Le sous-groupe des matrices diagonales est isomorphe au groupe des nombres complexes de module 1, et son dual à $\mathbf{Z}$. Les fonctions sphériques de la paires de Gelfand associée sont en bijection avec les caractères irréductibles de $S U(2)$. Ces caractères sont indexés par les entiers strictement positifs, et le caractère associé à l'entier $n$ est la fonction centrale sur $S U(2)$ telle que

$$
\varphi_{n}(g)=\frac{\sin n \theta}{n \sin \theta} ; \quad g=\left(\begin{array}{cc}
\mathrm{e}^{i \theta} & 0 \\
0 & \mathrm{e}^{-i \theta}
\end{array}\right) .
$$

On considère maintenant la fonction centrale $\varphi_{2}(g)=\frac{1}{2} \operatorname{Tr}(g)=\cos \theta$, c'est le caractère normalisé de la représentation irréductible de dimension 2 de $S U(2)$. Cette fonction engendre un semi-groupe multiplicatif $\varphi_{2}^{n}$. Il est facile de constater que la restriction de $\varphi_{2}$ à ce sous-groupe est la tranformée de Fourier de la loi de Bernoulli symétrique sur Z Z. Il s'ensuit que la restriction du semi-groupe associé à l'algèbre du sous-groupe diagonal est le semi-groupe de transition de la marche aléatoire simple sur $\mathbf{Z}$. Ses probabilités de transition sont donc

$$
p(k, k+1)=p(k, k-1)=\frac{1}{2} \quad k \in \mathbf{Z} .
$$


La restriction du semi-groupe à $M^{K}(G)$ donne un semi-groupe markovien sur les entiers qui peut se calculer en utilisant les formules de Clebsch-Gordan. On a en effet

$$
\varphi_{n} \varphi_{2}=\frac{n-1}{2 n} \varphi_{n-1}+\frac{n+1}{2 n} \varphi_{n+1}
$$

il s'ensuit que ce semi-groupe de probabilités de transition est donné par

$$
q(n, n+1)=\frac{n+1}{2 n} \quad q(n, n-1)=\frac{n-1}{2 n} \quad n \geq 1 .
$$

Les deux semi-groupes (3.1), (3.2) interviennent dans la démonstration du théorème de Pitman [17]. Le noyau d'entrelacement entre ces semi-groupes est facile à obtenir à partir des considérations précédentes. L'entier positif $n$ dans (3.2) paramètre une représentation irréductible de $S U(2)$, tandis que l'entier de (3.1) correspond à un caractère du sous-groupe diagonal. Le noyau d'entrelacement choisit uniformément un caractère parmi ceux qui interviennent dans la décomposition de la restriction de la représentation de dimension $n$ au sous-groupe diagonal. Ainsi ce noyau est donné par

$$
\Lambda(x, \mathrm{~d} y)=\sum_{k=0}^{x} \delta_{x-2 k}(\mathrm{~d} y)
$$

$x$ étant un entier positif et $y$ un entier relatif.

\subsection{Groupes d'automorphismes d'arbres}

Soit $q$ un entier (supérieur ou égal à 2). Soit $T_{q}$ un arbre homogène tel que le degré de chaque sommet soit $q+1$. C'est un espace métrique pour la distance naturelle donnée par la longueur d'un chemin minimal entre deux sommets. Soit $G$ le groupe des automorphismes de $T_{q}$, muni de la topologie de la convergence simple sur l'arbre, c'est un groupe localement compact, qui agit de façon doublement transitive sur l'arbre, et si $e$ est un sommet de $T_{q}$, le sous-groupe $K$ de $G$ des automorphismes fixant $e$ est un sous-groupe compact. D'après la section précédente, $(G, K)$ est donc une paire de Gelfand (voir [7]). Si $\phi$ est une fonction sur $G$, biinvariante par $K$, alors $\phi(g)$ ne dépend que de la distance $\mathrm{d}(e, g(e))$ dans l'arbre. On peut calculer les fonctions sphériques de type positif de la paire $(G, K)$. Elles sont indexées par les nombres réels $x \in\left[-\left(q^{1 / 2}+q^{-1 / 2}\right), q^{1 / 2}+q^{-1 / 2}\right]$, et sont données par la formule

$$
\varphi_{x}(g)=\frac{1}{q^{\frac{n}{2}}(q+1)}\left(q \frac{\lambda^{n+1}-\lambda^{-n-1}}{\lambda-\lambda^{-1}}-\frac{\lambda^{n-1}-\lambda^{-n+1}}{\lambda-\lambda^{-1}}\right):=q^{-n / 2} \sqrt{\frac{q}{q+1}} P_{n}(x)
$$

avec $n=\mathrm{d}(e, g(e))$ et $x=\lambda+\lambda^{-1}$, où $\lambda$ est un nombre complexe. Les formules étant symétriques en $\lambda$ et $\lambda^{-1}$, on choisira $\lambda$ de module inférieur à 1 .

On peut remarquer que $P_{n}(x)$ est un polynôme en $x$ qui vérifie une relation de récurrence à trois termes en $n$.

$$
x P_{n}(x)=P_{n+1}(x)+P_{n-1}(x) .
$$

Les $P_{n}$ forment en fait une suite de polynômes orthogonaux pour la mesure

$$
m_{q}(\mathrm{~d} x)=\frac{q(q+1)}{4 \pi} \frac{\sqrt{4-x^{2}}}{(q+1)^{2}-q x^{2}} \mathrm{~d} x
$$

sur l'intervalle $[-2,+2]$.

L'intervalle $\left[-\left(q^{1 / 2}+q^{-1 / 2}\right), q^{1 / 2}+q^{-1 / 2}\right]$ se décompose en $[-2,2]$, qui correspond à ce qu'on appelle la série principale, et en $\left[-\left(q^{1 / 2}+q^{-1 / 2}\right),-2[\cup] 2, q^{1 / 2}+q^{-1 / 2}\right]$, qui forme la série complémentaire. Lorsque $x$ parcourt 
la série principale, le paramètre $\lambda$ parcourt le cercle de rayon 1 , tandis que pour $x$ dans la série complémentaire, $\lambda$ parcourt $\left.]-1,-q^{-1 / 2}\right] \cup\left[q^{-1 / 2}, 1[\right.$ sur l'axe réel.

Il existe sur $G$ une fonction conditionnellement de type négatif privilégiée, qui est la fonction $\psi(g)=$ $-\mathrm{d}(e, g(e))$. Elle induit un semi-groupe de transformations complètement positives sur $M(G)$, que nous noterons $S_{t}$. Ce semi-groupe opère par multiplication sur les mesures, soit

$$
S_{t}(\mu)=\mathrm{e}^{t \psi} \mu
$$

Nous allons calculer sa restriction à deux algèbres commutatives bien choisies.

Soit $\tau \in G$ un élément tel que $\mathrm{d}\left(e, \tau^{n}(e)\right)=|n|$ pour tout $n \in \mathbf{Z}$. Un tel élément est facile à construire, $c f$. [4]. On note $T$ le groupe engendré par $\tau$, c'est un groupe libre à un générateur, isomorphe à $\mathbf{Z}$. Identifions donc $T$ avec $\mathbf{Z}$, alors le dual $\hat{T}$ de $T$ est le cercle unité de $\mathbf{C}$, et $\tau$ s'identifie avec la fonction $\omega \mapsto \omega: \hat{T} \rightarrow \mathbf{C}$. La restriction du semi-groupe $S_{t}$ à $M(T)$ est donnée par la formule

$$
\delta_{\tau^{n}} \rightarrow \mathrm{e}^{-|n| t} \delta_{\tau^{n}}
$$

soit en terme de fonctions sur le cercle unité

$$
\omega^{n} \rightarrow \mathrm{e}^{-|n| t} \omega^{n}
$$

elle correspond donc au noyau de Poisson

$$
Q_{t}(\xi, \mathrm{d} \omega)=\frac{1-\mathrm{e}^{-2 t}}{\left|\xi^{-1} \omega-\mathrm{e}^{-t}\right|^{2}} \mathrm{~d} \omega
$$

et le processus associé est le processus de Cauchy sur le cercle unité.

Examinons maintenant la restriction de $S_{t}$ à l'algèbre $M^{K}(G)$. On obtient un semi-groupe markovien sur l'intervalle $\left[-\left(q^{1 / 2}+q^{-1 / 2}\right), q^{1 / 2}+q^{-1 / 2}\right]$, dont on peut calculer les probabilités de transition grâce à l'expression explicite des fonctions sphériques. En effet, si on note $P_{t}(x, \mathrm{~d} y)$ ce noyau de probabilités, on aura

$$
\mathrm{e}^{-t \psi} \varphi_{x}=\int_{-\left(q^{1 / 2}+q^{-1 / 2}\right)}^{q^{1 / 2}+q^{-1 / 2}} \varphi_{y} P_{t}(x, \mathrm{~d} y)
$$

ce qui caractérise complètement la mesure de probabilités $P_{t}(x, \mathrm{~d} y)$. Le calcul peut s'effectuer selon la méthode suggérée dans [4]. On trouve alors l'expression suivante du semi-groupe. Soit $x$ dans la série complémentaire, $\left[-\left(q^{1 / 2}+q^{-1 / 2}\right),-2[\cup] 2, q^{1 / 2}+q^{-1 / 2}\right]$ correspondant à $\lambda \in\left[q^{-1 / 2}, 1\left[\right.\right.$. Si $\lambda \mathrm{e}^{t}<1$ on note $x(t)$ le point correspondant à $\lambda \mathrm{e}^{t}$ soit $x(t)=\lambda \mathrm{e}^{t}+\lambda^{-1} \mathrm{e}^{-t}$. Alors

$$
P_{t}(x, \mathrm{~d} y)=a_{t} \delta_{x(t)}+q_{t}(x, y) m(\mathrm{~d} y)
$$

où

et

$$
a_{t}=\frac{\left(\lambda \mathrm{e}^{t}-\lambda^{-1} \mathrm{e}^{-t}\right)\left(\lambda-q \lambda^{-1}\right)}{\left(\lambda \mathrm{e}^{t}-q \lambda^{-1} \mathrm{e}^{-t}\right)\left(\lambda+\lambda^{-1}\right)}
$$

$$
q_{t}(x, y)=\frac{q \lambda-\lambda^{-1}}{q+1}\left[\frac{q+1-y \lambda \mathrm{e}^{-t}}{1-y \lambda \mathrm{e}^{-t}+\lambda^{2} \mathrm{e}^{-2 t}}-\frac{\lambda-q \lambda^{-1}}{\lambda \mathrm{e}^{t}-q \lambda^{-1} \mathrm{e}^{-t}} \frac{q+1-y \lambda \mathrm{e}^{t}}{1-y \lambda \mathrm{e}^{t}+\lambda^{2} \mathrm{e}^{2 t}}\right] .
$$

Si $\lambda \mathrm{e}^{t} \geq 1$ ou si $x$ est dans la série principale, alors seule existe la partie continue du semi-groupe et on a

$$
P_{t}(x, \mathrm{~d} y)=q_{t}(x, y) m_{q}(\mathrm{~d} y)
$$


avec

$$
q_{t}(x, y)=\frac{1}{q+1}\left[\left(q \lambda-\lambda^{-1}\right) \frac{q+1-y \lambda \mathrm{e}^{-t}}{1-y \lambda \mathrm{e}^{-t}+\lambda^{2} \mathrm{e}^{-2 t}}-\left(q \lambda^{-1}-\lambda\right) \frac{q+1-y \lambda^{-1} \mathrm{e}^{-t}}{1-y \lambda^{-1} \mathrm{e}^{-t}+\lambda^{-2} \mathrm{e}^{-2 t}}\right] .
$$

Le processus gouverné par ce semi-groupe a le comportement suivant : s'il part d'un point de la série complémentaire, alors il suit une translation uniforme (dans la variable $\log \lambda$ ) avant de sauter à l'intérieur de la série principale. Le taux de saut est donné par la dérivée de la fonction $-a_{t}$. Une fois à l'intérieur de la série principale, il y reste et y effectue un processus de semi-groupe $q_{t}(x, y) m_{q}(\mathrm{~d} y)$.

Venons en maintenant à l'entrelacement entre ces processus. Pour le calculer, considérons un élément $\sigma \in G$ tel que $\mathrm{d}(e, \sigma(e))=n$. Pour un entier $n \geq 0$, posons

$$
\mu_{n}=\iint_{K \times K} \delta_{k_{1}} * \delta_{\sigma} * \delta_{k_{2}} \mathrm{~d} k_{1} \mathrm{~d} k_{2}
$$

Cette mesure est indépendante de $\sigma$, elle ne dépend que de $n$. Les mesures $\mu_{n} ; n \geq 1$ et $\mu_{0}=\delta_{e}$ engendrent l'algèbre $M^{K}(G)$ et vérifient la relation

$$
(q+1) \mu_{n} * \mu_{1}=\mu_{n-1}+q \mu_{n+1} ; n \geq 1
$$

qui exprime le fait géométrique simple que chaque sommet de l'arbre (hormis $e$ ) possède $q+1$ voisins dont $q$ sont plus éloignés de $e$ que lui, et un seul se trouve sur le plus court chemin menant à $e$. Il en résulte que les $\mu_{n}$ s'expriment sous forme de polynômes en $\mu_{1}$ (pour la convolution) plus exactement,

$$
\mu_{n}=q^{n / 2} P_{n}\left(\frac{q+1}{q^{1 / 2}} \mu_{1}\right)
$$

Cette égalité permet d'identifier l'algèbre $M^{K}(G)$ avec l'algèbre engendrée par $\mu_{1}$, et l'ensemble $\Omega$ correspond au spectre de l'élément $\frac{q+1}{q^{1 / 2}} \mu_{1}$ dans la $C^{*}$-algèbre engendrée par $M(G)$. Remarquons que ce spectre est plus grand que celui de $\mu_{1}$ agissant sur l'espace $L^{2}$ du groupe, car les représentations de la série complémentaire ne sont pas faiblement contenues dans la représentation régulière du groupe.

Ces considérations permettent de trouver le noyau d'entrelacement, et finalement le calcul donne le noyau

$$
\Lambda(x, \mathrm{~d} \omega)=\frac{q+1+q^{-1 / 2} \omega(q-x)}{1-q^{-1 / 2} x \omega+q^{-1} \omega^{2}} \mathrm{~d} \omega .
$$

\subsection{Groupes d'automorphismes d'espaces hyperboliques}

Nous allons maintenant considérer un exemple qui possède beaucoup de points communs avec celui des groupes d'automorphismes d'arbres, il s'agira des espaces hyperboliques. Nous nous bornerons ici au cas du quotient $S L_{2}(C) / S U(2)$ qui est un espace hyperbolique de dimension 3, mais les autres cas mériteraient qu'on s'y intéresse.

La décomposition polaire des matrices complexes $2 \times 2$ permet d'identifier l'espace hyperbolique $S L_{2}(C) / S U$ (2) avec l'ensemble des matrice complexes hermitiennes $2 \times 2$, définies positives, de déterminant 1 . Le groupe $S L_{2}(C)$ agit sur cet espace par automorphismes, suivant la formule $p \mapsto g p g^{*},\left(g \in S L_{2}(C)\right)$, le groupe d'isotropie de l'identité étant le sous-groupe $S U(2)$. L'action de $S L_{2}(C)$ est doublement transitive, et le couple $\left(S L_{2}(C), S U(2)\right)$ est un couple de Gelfand. Le spectre de ce couple de Gelfand est bien connu, et s'identifie de manière naturelle avec une partie du plan complexe, voir [10]. Il consiste en une partie principale, indexée par la droite complexe imaginaire pure $i \mathbf{R}$, et une partie supplémentaire, indexée par l'intervalle [-1,1]. Une fonction sphérique est complètement déterminée par sa restriction à l'ensemble des matrices de la forme

$$
g(x)=\left(\begin{array}{cc}
\mathrm{e}^{x} & 0 \\
0 & \mathrm{e}^{-x}
\end{array}\right)
$$


avec $x \geq 0$. Si $\omega \in i \mathbf{R} \cup[-1,1]$ la fonction sphérique correspondante est donnée

$$
\phi_{\omega}(g(x))=\frac{\sinh (\omega x)}{\omega \sinh (x)}
$$

avec

$$
\phi_{0}(g(x))=\frac{x}{\sinh (x)} .
$$

Nous allons considérer une fonction conditionnellement de type négatif remarquable sur $S L_{2}(C)$, biinvariante par $S U(2)$. Il s'agit, suivant la terminologie de [11], d'une forme quadratique généralisée. Cette fonction joue un rôle crucial dans la formule de Lévy-Khinchine sur l'espace hyperbolique. De telle fonctions peuvent s'obtenir pour tous les espaces hyperboliques de rang 1, et l'étude des processus stochastiques associés serait certainement intéressante. Pour construire cette fonction, on considére les fonctions conditionellement de type négatif $1-\varphi_{\omega}$, et on prend la limite

Le calcul donne

$$
\psi=\lim _{\omega \rightarrow 1} \frac{1-\varphi_{\omega}}{1-\omega}
$$

$$
\psi(g(x))=1-x \operatorname{coth}(x) .
$$

Le semi-groupe de fonctions de type positif engendré par $\psi$ peut se restreindre, comme précédemment, à deux sous-algèbres commutatives, d'une part les mesures portées par un sous-groupe commutatif, ici on prendra le sous-groupe des matrices diagonales de la forme $\left(\begin{array}{cc}\mathrm{e}^{t} & 0 \\ 0 & \mathrm{e}^{-t}\end{array}\right)$ avec $t \in \mathbf{R}$, d'autre part la sous-algèbre $M^{K}(G)$.

La restriction au sous-groupe diagonal donne un processus de Lévy $X_{t}$ d'exposant de Lévy $1-x \operatorname{coth} x$, tel que

$$
E\left[\exp \left(i x X_{t}\right)\right]=\exp (t(1-x \operatorname{coth} x) .
$$

La restriction à l'algèbre $M^{K}(G)$ donne un processus à valeurs dans $\Omega=i \mathbf{R} \cup[-1,1]$ et son semi-groupe de transition est déterminé par la formule

$$
\varphi_{\omega} \exp (t \psi)=\int_{\Omega} \varphi_{\xi} P_{t}(\omega, \mathrm{d} \xi)
$$

Trouver une formule explicite pour $P_{t}(\omega, \mathrm{d} \xi)$ impliquerait de donner la transformée de Fourier de la fonction $x \mapsto \exp t(1-x \operatorname{coth} x)$, ce qui ne semble pas possible en terme de fonctions spéciales connues. Toutefois on peut décrire sommairement le comportement du processus ayant ces probabilités de transition. En effet, on voit que si $\omega$ est dans la série complémentaire, alors le comportement à l'infini de la fonction sphérique $\varphi_{\omega}$ est $\varphi_{\omega}(x) \sim \frac{1}{\omega} \mathrm{e}^{(|\omega|-1) x}$. Si $\omega$ est dans la série principale, alors $\varphi_{\omega}(x)=0\left(\mathrm{e}^{-x}\right)$ On en déduit que si $\omega$ est dans la série complémentaire et si $t<1-|\omega|$, alors la décomposition en fonctions sphériques de la fonction $\varphi_{\omega} \exp (t \psi)$ admet une composante sur $\varphi_{\omega \pm t}$ (ici \pm est le signe de $\omega$ ). Plus précisément, la fonction $\varphi_{\omega} \exp (t \psi)-$ $\frac{|\omega|-t}{\omega} \varphi_{\omega \pm t}$ est $O\left(\mathrm{e}^{-x}\right)$ à l'infini, donc se décompose purement sur la série principale. Le processus admet donc un comportement semblable à celui de la section précédente : s'il commence dans la série complémentaire, alors il suit un mouvement de translation uniforme avant de sauter dans la série principale. Une fois arrivé dans la série principale, il y reste et y décrit un processus de saut. On peut maintenant déduire des considérations générales du début l'existence d'un noyau d'entrelacement entre ce processus et le processus de Lévy dont l'exposant de Lévy est $1-x \operatorname{coth} x$. Toutefois l'absence de formule explicite pour la transformée de Fourier de $\exp (t(x \operatorname{coth} x-1))$ ne permet pas d'en donner une expression exacte.

Pour terminer je voudrais signaler une relation mystérieuse (pour moi) entre ce processus et le mouvement Brownien sur le groupe d'Heisenberg. L'intersection des séries principale et complémentaire de l'ensemble $\Omega$ correspond à la fonction sphérique $\varphi_{0}(g(x))=\frac{x}{\sinh (x)}$. Si nous partons de ce point, la loi du processus à l'instant $t$ est déterminée par la décomposition de la fonction de type positif

$$
\frac{x}{\sinh (x)} \exp (t(1-x \operatorname{coth} x))
$$


en fonctions sphériques. Il se trouve que cette même fonction (3.3) apparaît dans le calcul du noyau de la chaleur correspondant au Laplacien hypoelliptique sur le groupe d'Heisenberg, c'est la formule de l'aire de Paul Lévy, $c f$. [12]. Il serait intéressant d'avoir une explication de cette coïncidence.

\section{RÉFÉRENCES}

[1] P. Biane, Quantum random walk on the dual of SU(n). Probab. Theory Relat. Fields 89 (1991) 117-129.

[2] P. Biane, Minuscule weights and random walks on lattices, Quantum probability and related topics, QP-PQ VII. World Scientific Publishing, River Edge, NJ (1992) 51-65.

[3] P. Biane, Intertwining of Markov semi-groups, some examples. in Séminaire de Probabilités XXIX. Lecture Notes in Math. 1613, Springer, Berlin (1995) 30-36.

[4] P. Biane, Quantum Markov processes and group representations, Quantum probability communications, $Q P-P Q \mathbf{X}$. World Scientific Publishing, River Edge, NJ (1998) 53-72.

[5] P. Biane, Le théorème de Pitman, le groupe quantique $\mathrm{SU}_{q}(2)$, et une question de P.A. Meyer, In memoriam Paul-André Meyer, in Séminaire de Probabilités XXXIX. Lecture Notes in Math. 1874, Springer, Berlin (2006) 61-75.

[6] P. Carmona, F. Petit and M. Yor, Beta-gamma random variables and intertwining relations between certain Markov processes. Rev. Mat. Iberoamericana 14 (1998) 311-367.

[7] F.M. Choucroun, Analyse harmonique des groupes d'automorphismes d'arbres de Bruhat-Tits. Mém. Soc. Math. France (N.S.) $58(1994)$

[8] A. Connes, Noncommutative geometry. Academic Press, Inc., San Diego, CA (1994).

[9] J. Dubédat, Reflected planar Brownian motions, intertwining relations and crossing probabilities. Ann. Inst. H. Poincaré Probab. Statist. 40 (2004) 539-552.

[10] J. Faraut, Analyse sur les paires de Gelfand, in Analyse harmonique. Les Cours du CIMPA (1982).

[11] J. Faraut and K. Harzallah, Distances hilbertiennes invariantes sur un espace homogène. Ann. Inst. Fourier (Grenoble) 24 (1974) $171-217$.

[12] B. Gaveau, Principe de moindre action, propagation de la chaleur et estimées sous-elliptiques sur certains groupes nilpotents. Acta Math. 139 (1977) 95-153.

[13] F. Hirsch and M. Yor, Fractional intertwinings between two Markov semi-groups. Potential Anal. 31 (2009) $133-146$.

[14] H. Matsumoto and M. Yor, An analogue of Pitman's $2 M-X$ theorem for exponential Wiener functionals. Part I. A timeinversion approach. Nagoya Math. J. 159 (2000) 125-166.

[15] N. O'Connell, Directed polymers and the quantum Toda lattice. arXiv:0910.0069

[16] K.R. Parthasarathy, An introduction to quantum stochastic calculus. Monographs Math. 85, Birkhäuser Verlag, Basel (1992).

[17] J.W. Pitman, One-dimensional Brownian motion and the three-dimensional Bessel process. Adv. Appl. Probab. 7 (1975) 511-526. 